\title{
Online optical refractive index measurement in research reactor core
}

\author{
Gary Fourneau $^{1}$, Marion Agoyan ${ }^{1,2}$, Guy Chemol $^{1}$, Ayoub Ladaci ${ }^{1}$, Hicham Maskrot ${ }^{1}$, \\ Christophe Destouches ${ }^{3}$, Damien Fourmentel ${ }^{3}$, Sylvain Girard ${ }^{2}$, Aziz Boukenter ${ }^{2}$ \\ ${ }^{1}$ CEA, DES/ISAS/DPC/SEARS/LISL, Saclay, France \\ ${ }^{2}$ Université Jean Monnet, CNRS, Graduate School Optics Institute, Laboratoire Hubert Curien UMR \\ 5516, Saint-Etienne, France \\ ${ }^{3} \mathrm{CEA}, \mathrm{DES} / \mathrm{IRESNE} / \mathrm{DER} / \mathrm{SPESI}$, Cadarache, France \\ gary.fourneau@cea.fr
}

\begin{abstract}
There is a growing interest in fiber optic measurements for applications in radiation environments. Optical fiber sensors and diagnostics can monitor many parameters of interest inside a research reactor core. For some applications, fiber optics are combined with an optical system that collects or focuses the light beam. The RadiationInduced-Refractive-Index-Change (RIRIC) of the used glasses appears then as major phenomenon as it is a determining value for the sensor optical function. In the framework of the development of a radiation hardened confocal chromatic sensor, we implemented an on-line refractive index measuring device in order to test in a reactor core various glasses, candidates to be implemented into the sensor. The measurement relies on interferometry, which is a challenge because of the small volume available, the impossibility to make optical adjustments once installed, and the high temperature of operation. Precisely, the quantity retrieved is the optical path (product of the length $L$ by the optical refractive index $n$ ), when $L$ is well known, we can deduce $n$. But under high neutron fluence, some variation in density can be observed. The targeted online measurement of the refractive index therefore becomes an optical path measurement. We will present the device, the principle of measurement, and the first results of some index change measurement, produced by a temperature ramp from $20{ }^{\circ} \mathrm{C}$ and $350{ }^{\circ} \mathrm{C}$. We have obtained original data for most of the candidate glasses used to design the optical system.

Keywords -Optical fiber sensor, Radiation-InducedAttenuation, Interferometry, Radiation-Induced-RefractiveIndex-Change, Interferometric measurement, Rad-hard Glasses, High neutron fluence.
\end{abstract}

\section{INTRODUCTION}

$\mathrm{T}$ HIS work is made in the framework of the Instrumentation for Nuclear Applications (INSNU) project, aiming to develop instrumentation and sensors for experiments in the Jules Horowitz Reactor (Cadarache, France). Among other sensors, we develop optical ones and particularly a confocal chromatic sensor [1]. While on some measurements on intrinsic optical fiber sensor, the fiber is the only passive component exposed to the harsh environment constraint, in the case of extrinsic sensors, such as the confocal chromatic [1], additional components are involved and need to be characterized. In our case, the sensor relies on the use of an optical system made of several glass components, thus leading to some issues caused by the influence of radiation and high temperatures on their performances. As for fiber optics in such reactor environment $[2,3]$, the two main radiation effects on bulk glasses are the Radiation Induced Attenuation (RIA) and the density change leading to dimensional change and Radiation Induced Refractive Index Change (RIRIC). Unfortunately, little data is available in the literature, a few studies are available but without the neutron contribution and at much lower gamma dose levels, they are then not representative of what is expected in an in-pile experiment [4].

In order to collect data on glass darkening and refractive index change we will design and perform in 2022 two specific experiments inside the TESCA (Test in Capsule) irradiation devoted to fiber optic sensor (FOS) -in Belgium Reactor 2. The two small measurement systems aim to provide online measurements, allowing to characterize both transient and permanent effects. First systems is designed to collect RIA data while the second is devoted to refractive index change measurements, more precisely for optical path measurement $\mathrm{n} x$ L. We focus here on the $n \times \mathrm{L}$ measurement using a white light interferometry approach.

The specifications expected for the system are as follows: Mechanical diameter : $9 \mathrm{~mm}$

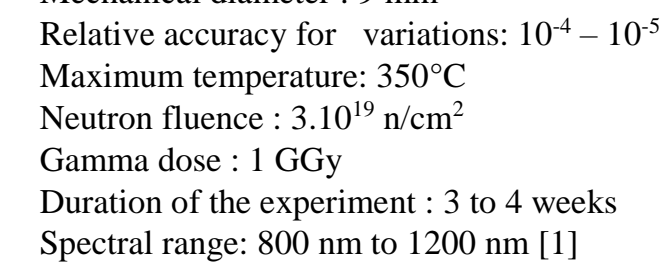

Of course, it would have been preferable to measure separately $\mathrm{n}$ and L. But with such constraints, the usual techniques making it possible to measure $\mathrm{n}$ and $\mathrm{L}$ separately cannot be implemented inside a reactor core. We are also preparing additional samples for measurements to be done before and after irradiation on glass samples to try to decorrelate between the variations of $n$ and $L$.

In order to collect as much information as possible, the $\mathrm{n} \times \mathrm{L}$ measuring device is expected be able to:

Test various types of glass.

- Measure the optical path variation induced by 
radiations at a constant temperature of irradiation.

- Operate at several wavelengths within the spectral domain of interest

- $\quad$ Also (out of pile experiment) measure the variation of the optical path induced by the temperature only (thermal dilatation and impact on the refractive index).

The optical fiber will carry the probe beam and will collect and carry back to the optical signal to the conditioner.

To perform such a measurement, we monitor the evolution of the channeled spectrum resulting from the interference between the two parallel faces of a cylindrical glass sample. In this paper, we present the architecture of the measurement device and its operation principle. We will present the preliminary results obtained in the laboratory varying the temperature only.

\section{THE OPTICAL PATH MEASUREMENT DEVICE, SIGNAL CONDITIONER AND SIGNAL PROCESSING}

\section{A. Experimental setup}

To respond to our needs, we developed an opto-mechanical device using a Single Mode (SM) optical fiber. Fig. 1 shows the developed setup.

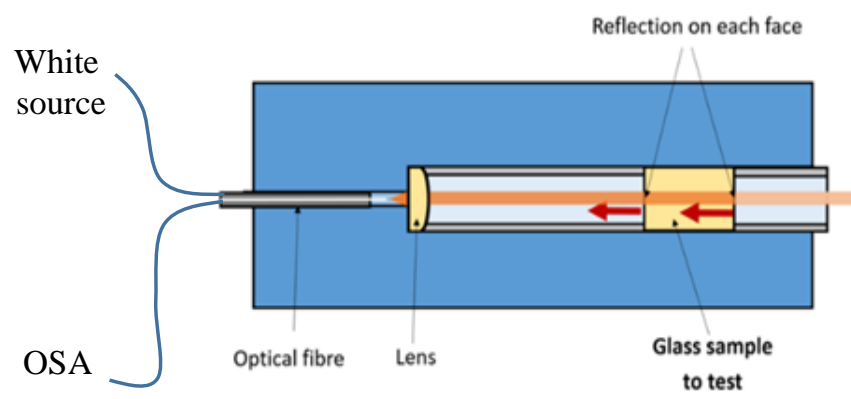

Figure 1: Schematic diagram of the interferometric setup to measure refractive index or optical path change under radiative environment.

The light comes from a white supercontinuum source, which has a nearly constant intensity from $800 \mathrm{~nm}$ to $1500 \mathrm{~nm}$ (except a peak around the pump signal at $1064 \mathrm{~nm}$ ); this allows us to use a single source to observe the wide spectral domain of interest. The light beam coming out of the fiber goes through a lens to collimate it. A part of the beam is then reflected on each face of the glass sample under test. These reflected rays interfere and are reinjected inside the fiber. Finally, the light is sent to an Optical Spectrum Analyzer (OSA) which allows observing the channeled spectrum (Fig. 2) resulting from the interference. The theoretical shape for such spectrum is given by the following formula, it is then needed to process the measured signal in order to extract the $\mathrm{n} \times \mathrm{L}$ value:

$$
I(\lambda, T)=I_{0}(\lambda)\left[A+B \cos \left(2 \pi\left(\frac{2(n(\lambda, T) \times L(T))}{\lambda}\right)+\varphi_{0}\right)\right]
$$

where $I_{0}(\lambda)$ is the source's intensity spectrum, A and B are constants, $\mathrm{n}$ is the refractive index of the glass sample and $\mathrm{L}$ its length. $\lambda$ is the wavelength and $\mathrm{T}$ the temperature.

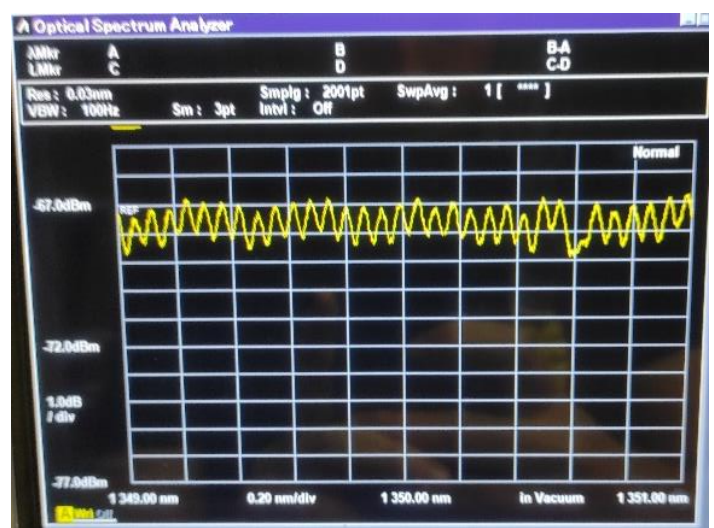

Figure 2: Example of a channeled spectrum obtained from the device.

\section{B. Improved setup: the double interferometer}

The Interfringe (If) of the channeled spectrum is :

$$
\text { If } \sim \frac{\lambda^{2}}{2 . n . L}
$$

Our samples are approximately $10 \mathrm{~mm}$ long. With $\mathrm{n} \sim 1.5$ and $\lambda=1 \mu \mathrm{m}$, (2) gives: If $\sim 30 \mathrm{pm}$ which is roughly the minimum spectral resolution available with our spectrometer. Working with an If in the same range than the spectral resolution affects the modulation, especially at lower wavelengths.

This led us to design a more complex setup than the one illustrated in Fig.1, that consists in two interferometers in series. This architecture allows also increasing the accuracy of the measurement. The first interferometer is the previously described small measurement system while the second one is made of a cavity in the air with a length Lc close to $n \times L$. The resulting spectrum corresponds to the product of the channeled spectrum of both cavities taken separately:

$$
\begin{gathered}
I_{\text {tot }}(\lambda, T)=I_{1}(\lambda, T) \times I_{2}(\lambda, T) \\
I(\lambda, T) \approx \cos \left(2 \pi\left(\frac{2 n(\lambda, T) \times L(T)}{\lambda}\right)\right) \times \cos \left(2 \pi\left(\frac{2 L c}{\lambda}\right)\right)
\end{gathered}
$$

With a little bit of trigonometry we can show that this signal is composed of four main frequencies:

$$
\begin{array}{ll}
- & 2 n \times L \\
- & 2 L c \\
- & 2(n \times L+L c) \\
- & 2|n \times L-L c|
\end{array}
$$

$\mathrm{n} \times \mathrm{L}$ and Lc both give high frequency signals, affected by the limited resolution of the OSA, which also does not permit to observe at all the signal with the $(n \times L+L c)$ frequency.

However, since $n \times L$ and Lc are about the same length, the frequency last remaining in our signal is rather low and easy to measure accurately. This frequency only depends on the difference of length between the two cavities. If we know the exact length of the second cavity, we have access to the length of the optical path measured with a far better accuracy than with our first setup.

\section{Signal processing principle}

In order to have access to the optical length's value, we have to process the channeled spectrum. Firstly, let us consider that $\mathrm{n}$ is mainly independent of the wavelength. In that case, the 
spectrum is a periodic function of $\frac{1}{\lambda}$ with a frequency: $2 n \times L$. To determine this frequency, we apply a Fast Fourier Transform (FFT) on the acquire signal and we search for a peak near the expected value of $2 \mathrm{n} \times \mathrm{L}$, the abscissa of this peak gives us the exact value of the optical path measured.

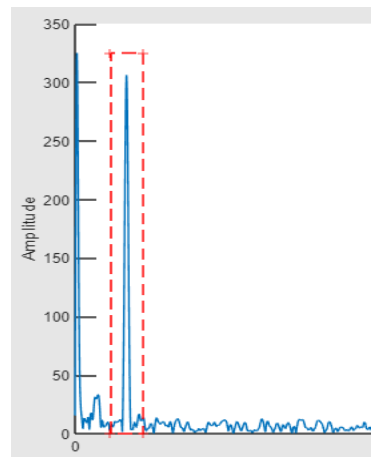

Figure 3: Example of a FFT applied to an experimental signal. The red rectangle corresponds to the domain where we expect to find a peak beforehand. The X-axis corresponds to the optical length measured

That is what happens with an extrinsic Fabry-Perot interferometer [5]. However, doing this way, experimental results are shifted by $150 \mu \mathrm{m}$ to $250 \mu \mathrm{m}$. In fact, the glasses' refractive indexes depend on the wavelength. The chromaticity is responsible for the error observed. With this method, we do not have access to the refractive index but to the group index of the tested glass, the actual frequency retrieved by the FFT is not $n\left(\lambda_{0}\right) \times L$ as expected but rather :

$$
n^{\prime} \times L=\left(n\left(\lambda_{0}\right)-\frac{d n}{d \lambda}\left(\lambda_{0}\right) \cdot \lambda_{0}\right) \times L
$$

It is then needed to add a correction to the initial value obtained by reading the peak's abscissa:

$$
n\left(\lambda_{0}\right)=n^{\prime}+\frac{d n}{d \lambda}\left(\lambda_{0}\right) \cdot \lambda_{0}
$$

This mean that we need to know the value of $\frac{d n}{d \lambda}\left(\lambda_{0}\right)$ beforehand or we assume that this value does not vary with the temperature or radiations and we confirm it afterwards.

We have checked that using this signal processing and the correction on a simulated spectrum with a given glass and length, we get $\mathrm{n} \times \mathrm{L}$ with a relative error of $10^{-6}$ which may be caused by computing errors.

We also developed another method for processing the signal that consists of following the evolution of a single peak of the channeled spectrum to determine the variations of optical path [6]. While much more accurate in theory, this solution is actually not adapted to the TESCA experiment as it requires small variation between too acquisitions of the peak position to ensured that the peak observed has not been replaced by another one. We will test several samples in parallel, with therefore some delay between two acquisitions. Operation in a reactor core lacks stability to allow this measurement; which on the other hand permitted us to measure the stability of the compensating interferometer.

\section{RESULTS FOR VARIATION OF N $\times$ L WITH THE TEMPERATURE ONLY}

Obtaining and maintaining the optical adjustment which gives the interferometric signal is a difficult task, especially with the disturbances due to the increase in temperature. Once that was guaranteed, we measured the variation of the refractive index with the temperature of various glasses. Here, we know the value $\mathrm{L}(\mathrm{T})$, thanks to datasheets giving the thermal dilatation up to high temperatures, therefore by dividing the $\mathrm{n}(\mathrm{T}) \times \mathrm{L}(\mathrm{T})$ product by $\mathrm{L}(\mathrm{T})$, we obtained $\mathrm{n}(\mathrm{T})$. Fig. 6 display the result obtained for SF6G05 at three wavelengths.

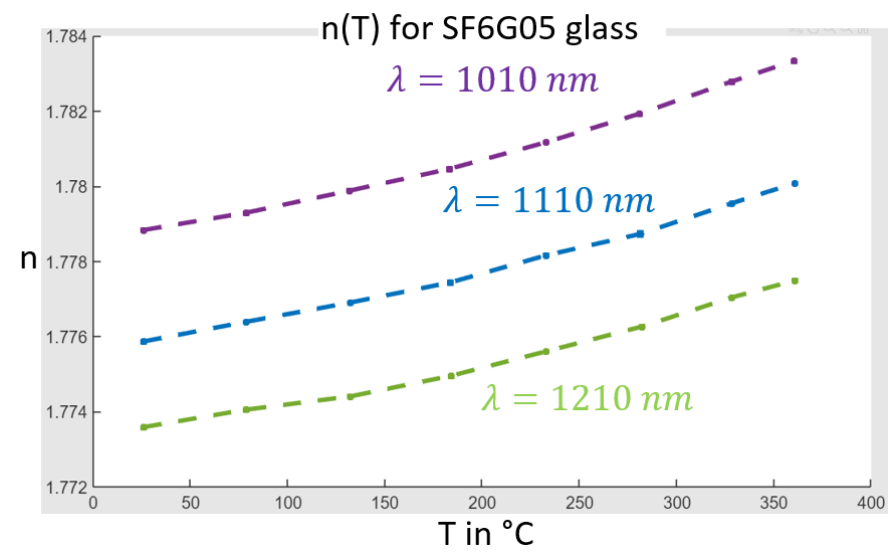

Figure $4: n(T)$ obtained for SF6G05 glass using our setup and signal processing

We observe a difference of nearly $10^{-3}$ for absolute values of the refractive index at low temperature compared to the theoretical values provided by the glassmaker Schott. For other glasses, the offset is in the same range (few 10-3). Possible reasons for these errors are some differences due to different glass batches, error in measuring the glass sample or the second cavity lengths. These errors will remain constant in the planned irradiation and should not affect our ability to measure $n \times L$ variations under irradiation.

\section{CONCLUSION}

In order to develop a rad-hard confocal chromatic sensor, data on the behavior of glasses under radiation are expected to result from the TESCA irradiation (2022, in the BR2 research reactor). We have developed a smart miniature device in order to measure by interferometry the variations of the optical path $\mathrm{n} \times \mathrm{L}$ of some glass samples during in-core irradiation. This device will be soon set in the research reactor's core for online measurements.

Today, the robustness of the device has been tested by measuring the $\mathrm{n} \times \mathrm{L}$ variations with temperature, giving access to $\mathrm{n}(\mathrm{T})$ up to $350^{\circ} \mathrm{C}$, data not currently available in the literature for most glasses. Work is still in progress to consolidate the accuracy of our setup and remains necessary to design an optical system able to operate at high temperature. 


\section{REFERENCES}

[1] M. Agoyan et al, « Confocal chromatic sensor for displacement monitoring in research reactor », Animma 2021.

[2] S. Girard et al., "Overview of radiation induced point defects in silicabased optical fibers," Reviews in Physics, 2019, doi: https://doi.org/10.1016/j.revip.2019.100032

[3] L. Remy, G. Cheymol, A. Gusarov, A. Morana, E. Marin and S. Girard, "Compaction in Optical Fibres and Fibre Bragg Gratings Under Nuclear Reactor High Neutron and Gamma Fluence," in IEEE Transactions on Nuclear Science, vol. 63, no. 4, pp. 2317-2322, Aug. 2016, doi: 10.1109/TNS.2016.2570948.

[4] C. Muller, T. Lépine, T. Allanche, A. Boukenter, P. Paillet, S. Girard, and Y. Ouerdane "Potential performance loss and compensation techniques of a lens under ionizing radiations", Proc. SPIE 10690, Optical Design and Engineering VII, 106900T (5 June 2018)

[5] G. Cheymol, A. Verneuil, P. Grange, H. Maskrot and C. Destouches, "HighTemperature Measurements With a Fabry-Perot Extensometer," in IEEE Transactions on Nuclear Science, vol. 67, no. 4, pp. 552-558, April 2020, doi: 10.1109/TNS.2020.2975707.

[6] C.Z. Tan, J. Arndt, "Temperature dependence of refractive index of glassy $\mathrm{SiO} 2$ in the infrared wavelength range", Journal of Physics and Chemistry of Solids, vol. 61, pp. 1315-1320, Nov. 1999 